ARTICLE

https://doi.org/10.1038/s41467-019-13540-4

\title{
The autophagy receptor p62/SQST-1 promotes proteostasis and longevity in $C$. elegans by inducing autophagy
}

Caroline Kumsta ${ }^{1 \star}$, Jessica T. Chang ${ }^{1}$, Reina Lee ${ }^{1}$, Ee Phie Tan ${ }^{1}$, Yongzhi Yang ${ }^{1}$, Rute Loureiro ${ }^{2}$, Elizabeth H. Choy ${ }^{1}$, Shaun H.Y. Lim (1) 1, Isabel Saez², Alexander Springhorn², Thorsten Hoppe (D) ${ }^{2}$, David Vilchez (iD ${ }^{2} \&$ Malene Hansen ${ }^{1 *}$

Autophagy can degrade cargos with the help of selective autophagy receptors such as p62/ SQSTM1, which facilitates the degradation of ubiquitinated cargo. While the process of autophagy has been linked to aging, the impact of selective autophagy in lifespan regulation remains unclear. We have recently shown in Caenorhabditis elegans that transcript levels of sqst-1/p62 increase upon a hormetic heat shock, suggesting a role of SQST-1/p62 in stress response and aging. Here, we find that sqst-1/p62 is required for hormetic benefits of heat shock, including longevity, improved neuronal proteostasis, and autophagy induction. Furthermore, overexpression of SQST-1/p62 is sufficient to induce autophagy in distinct tissues, extend lifespan, and improve the fitness of mutants with defects in proteostasis in an autophagy-dependent manner. Collectively, these findings illustrate that increased expression of a selective autophagy receptor is sufficient to induce autophagy, enhance proteostasis and extend longevity, and demonstrate an important role for sqst-1/p62 in proteotoxic stress responses.

\footnotetext{
${ }^{1}$ Sanford Burnham Prebys Medical Discovery Institute, Development, Aging and Regeneration Program, 10901 North Torrey Pines Road, La Jolla, CA 92037, USA. ${ }^{2}$ Institute for Genetics and Cologne Excellence Cluster for Cellular Stress Responses in Aging-Associated Diseases (CECAD), University of Cologne, Joseph Stelzmann Strasse 26, 50931 Cologne, Germany. *email: ckumsta@sbpdiscovery.org; mhansen@sbpdiscovery.org
} 
$\mathrm{M}$ acroautophagy (hereafter called autophagy) facilitates degradation and recycling of cytosolic components, referred to as cargo, in response to nutrient deprivation or other stressors. Autophagy is initiated by the nucleation of a double membrane, which forms the phagophore. As the phagophore expands, it begins to sequester cytosolic cargo into the growing vesicle. Upon completion, the autophagosome or amphisome (formed by fusion with vesicles from the endolysosomal compartments) then fuse with acidic lysosomes, resulting in the degradation of the sequestered content by hydrolases. Autophagy is essential for survival, development, and organismal homeostasis ${ }^{1}$. It occurs at low levels under basal conditions, whereas developmental stimuli or cellular stress, including starvation and heat shock (HS), can induce autophagy ${ }^{1,2}$. Furthermore, autophagy can protect against pathologies, including neurodegeneration and aging ${ }^{3}$. The regulation of autophagy with age is incompletely understood, but several lines of evidence suggest that autophagy declines with age ${ }^{3}$. Conversely, autophagy genes are essential for lifespan extension in distinct longevity paradigms in S. cerevisiae, C. elegans, and Drosophila (for review see ref. ${ }^{3}$ ). While these observations demonstrate a link between autophagy and aging, it remains unclear how the autophagy process affects longevity and healthspan.

Autophagy was originally described as a 'bulk' turnover process, in which cytosolic components are indiscriminately recycled to provide amino acids and other building blocks during nutrient deprivation and cellular stress ${ }^{4}$. Emerging evidence indicates that selective types of autophagy degrade specific and possibly damaged cytosolic components in a tightly regulated manner. During selective autophagy, autophagosomes recruit specific types of cargo, including mitochondria and protein aggregates, through the action of autophagy receptors that connect the autophagosome to the cargo ${ }^{4}$. The binding of the autophagy receptors is facilitated by Atg8/LC3/GABARAP family proteins, which are proteolytically cleaved and conjugated with phosphatidylethanolamine and incorporated into the forming autophagosomal membranes ${ }^{4}$. The selective autophagic degradation of ubiquitinated protein aggregates, termed aggrephagy, can be facilitated by autophagy receptor p62/SQSTM1 (hereafter referred to as p62). p62 has several conserved domains that are important for its function in autophagy. The C-terminal ubiquitin-associated (UBA) domain enables p62 to bind to ubiquitinated proteins ${ }^{4}$. The LC3-interacting region (LIR domain) allows binding of p62 to Atg8/LC3/GABARAP family members, and thus facilitates degradation of ubiquitinated protein $\mathrm{cargo}^{5,6}$. p62-mediated aggrephagy also requires the Phox-and-Bem1 (PB1) domain of $\mathrm{p} 62$, which allows its self-oligomerization and strengthens binding to the Atg8/LC3-containing autophagic membranes ${ }^{7}$.

The degradation of ubiquitinated proteins can occur via autophagy as well as the ubiquitin-proteasome system (UPS), and p62 has been implicated in both processes; i.e., as a selective autophagy receptor, and through the delivery of ubiquitinated proteins for degradation to the proteasome ${ }^{8-12}$. p62 is constantly being degraded by autophagy ${ }^{5}$, and inhibition of autophagy leads to the accumulation of p62 aggregates in mice 7 Drosophila ${ }^{13}$, and C. elegans ${ }^{14}$. These ubiquitin-positive aggregates are significantly reduced in autophagy-deficient mice and flies when p62 is deleted $^{13,15}$, indicating a role for p62 in aggregate formation. Furthermore, p62 has been found associated with ubiquitinpositive aggregates observed in neurodegenerative-disease models, such as Huntington's and Alzheimer's disease ${ }^{13}$. The biological relevance of aggregate formation is still under debate and may depend on the cellular context and the size of the aggregates. To this end, it has been suggested that larger aggregates are less toxic to the cell than smaller aggregates dispersed throughout the cell $^{16-18}$. While p62 overexpression can enhance protein aggregation, overexpression of $\mathrm{p} 62$ has protective effects on cell survival $^{5}$ and p62 has an important role in preventing neurodegenerative diseases ${ }^{10,12}$, although the exact protective mechanisms are still unclear.

Consistent with a key role in age-related disease, mice deficient in p62 have reduced lifespan, increased oxidative stress ${ }^{19}$, synaptic deficiencies, and memory impairment ${ }^{20}$, albeit neither significant neurodegeneration nor aggregation formation are observed in neurons of these mice ${ }^{15}$. Older $p 62^{-/-}$mice, however, accumulate insoluble ubiquitinated proteins in the brain $^{21}$. Similar to mice, Drosophila expressing mutant $\operatorname{Ref}(2) \mathrm{P} / \mathrm{p} 62$, which lacks either the PB1 or the UBA domain, are short-lived, have impaired movement, and mitochondrial defects ${ }^{22}$. In contrast, in C. elegans, mutations in the 062 ortholog sqst-1 cause no lifespan defects ${ }^{23,24}$, indicating a limited requirement for sqst-1/p62 (hereafter called sqst-1) under basal conditions in the nematode.

The expression levels of $p 62$ have been shown to decline with age in mice ${ }^{19}$, and reduced expression of $p 62$ correlates with agerelated neurodegenerative diseases in humans ${ }^{25,26}$. Notably, we recently reported that sqst-1 mRNA levels are markedly increased upon heat stress in C. elegans ${ }^{2}$, prompting the hypothesis that SQST-1 may play a role in the heat-shock response, in which heat-shock proteins and molecular chaperones are rapidly and transiently induced to ameliorate the deleterious effects of heat stress ${ }^{27}$. We further reported that exposure of $C$. elegans to a hormetic HS, i.e., a short stimulus with increased temperature that would be lethal at prolonged exposure, not only increases stress resistance and longevity, but also improves proteostasis in an autophagy-dependent manner ${ }^{2}$. Since emerging evidence suggests that the degradation of specific cargos by selective autophagy is important for maintaining health ${ }^{3}$, we investigated the role of SQST-1 in hormetic HS, lifespan, and proteostasis. Here we demonstrate that sqst-1 is required for autophagy induction as well as organismal benefits conferred by a hormetic HS. Furthermore, we show that overexpression of full-length SQST-1, but not truncated SQST-1 lacking the UBA domain, is sufficient to increase longevity in C. elegans. SQST-1 overexpression leads to tissue-specific induction of autophagy and decreases aggregation of expanded PolyQ proteins as well as temperature-sensitive protein-folding mutants in an autophagydependent manner. These observations illustrate that overexpression of a selective autophagy receptor is sufficient to induce autophagy and enhance longevity and proteostasis. As p62 plays an important role in many age-related diseases, our findings highlight potential therapeutic opportunities in inducing p62mediated selective autophagy.

\section{Results}

sqst-1 regulates basal autophagy in a tissue-specific manner. A hormetic heat shock (HS) early in life of C. elegans induces autophagy and increases the transcription of autophagy-related genes, including $s q s t-1^{2}$. HS causes proteotoxic stress, which is accompanied by protein unfolding and can ultimately lead to protein aggregation ${ }^{28,29}$. Protein aggregates and ubiquitinated protein conjugates accumulate upon a $\mathrm{HS}$ of $1 \mathrm{~h}$ at $36^{\circ} \mathrm{C}$ in C. elegans (Supplementary Fig. 1a, b), as observed in human melanoma cells ${ }^{30}$. Since ubiquitinated proteins can be degraded by selective autophagy via the autophagy receptor $\mathrm{p} 62^{5}$, and a hormetic HS induces an increase of autophagosomes in C. elegans ${ }^{2}$, we asked if sqst-1 could play a role in the regulation of autophagy under heat-stress conditions. To this end, we first examined whether reduction of sqst-1 function would lead to autophagy defects under basal conditions. While SQST-1 protein expression appears limited to the pharynx and nerve-ring 
neurons under basal conditions, sqst-1 is transcriptionally expressed in nearly all tissues in C. elegans ${ }^{14,31,32}$. Therefore, we monitored autophagy in nerve-ring neurons, intestine, body-wall muscle, and pharynx of sqst-1(ok2892) mutants, which expresses a truncated loss-of-function SQST-1 protein ${ }^{24,33}$, and in a CRISPR-engineered, full-length deletion of sqst-1 (sqst-1(syb764)) expressing GFP-tagged LGG-1/Atg8 $8^{34-36}$. This autophagy reporter visualizes autophagic structures (i.e., pre-autophagosomal structures, autophagosomes, and amphisomes) as fluorescent punctae.

Both sqst-1 mutants showed a decrease in the number of GFP::LGG-1/Atg8-positive punctae in nerve-ring neurons (Fig. 1a and Supplementary Fig. 2a, b), whereas the number of GFP::LGG-1/Atg8-positive punctae was unchanged in the intestine, body-wall muscle, or pharynx (Fig. $1 \mathrm{~b}-\mathrm{d}$ and Supplementary Fig. 2c-h) [For hypodermal seam cells, see Supplementary Fig. 2i, j]. Concomitant analysis of a lipidationdeficient GFP::LGG-1(G116A) mutant ${ }^{37}$ confirmed that LGG-1/Atg8-positive structures were likely to represent autophagosomes in these four major tissues (Supplementary Fig. 2). Since the two sqst-1 mutants displayed the same autophagy phenotypes and were not compromised in the healthspan measures we investigated, i.e., movement, pharyngeal pumping, or progeny production (Supplementary Fig. 3), we used the sqst-1(ok2892) mutants in our subsequent analyses, unless stated otherwise.

Monitoring the number of GFP::LGG-1/Atg8-positive punctae does not conclusively inform on autophagic activity ${ }^{38}$. Therefore, we also performed autophagy-flux assays in sqst-1(ok2892) mutants by injecting the autophagy inhibitor bafilomycin A1 (BafA), which blocks autophagosomal turnover ${ }^{39,40}$. A change in the number of GFP::LGG-1/Atg8-positive punctae upon BafA addition indicates that autophagy is active, whereas no change indicates that the cell/tissue is experiencing a block in autophagy. Notably, BafA treatment did not increase the number of GFP::LGG-1/Atg8-positive punctae in the nerve-ring neurons of sqst-1(ok2892) mutants, which had very low numbers of autophagosomes under basal conditions (Fig. 1a), Taken together, these findings indicate that sqst-1 is required for autophagosome formation in neurons, as observed in $\mathrm{HeLa}$ cells ${ }^{7}$, rather than increasing the rate of autophagosomal turnover. In contrast, the intestine (Fig. 1b), body-wall muscles (Fig. 1c), as well as the pharynx (Fig. 1d) showed a clear increase in the number of GFP::LGG-1/Atg8-positive punctae after BafA injection, consistent with autophagic turnover under basal conditions in nonneuronal tissues. In sum, these results indicate that sqst-1(ok2892) animals display a tissue-specific block in autophagy in nerve-ring neurons.

sqst-1 is required for autophagy induction upon heat shock. To determine whether sqst-1 could play a role in the regulation of autophagy in response to heat stress, we next asked whether a hormetic HS would induce autophagy in different tissues of sqst-1 (ok2892) mutants. In contrast to WT animals ${ }^{2}$, exposure of sqst-1 (ok2892) mutants to a hormetic HS failed to induce autophagosome accumulation in nerve-ring neurons (Fig. 1e), intestine (Fig. 1f), body-wall muscle (Fig. 1g), and pharynx (Fig. 1h), indicating that $s q s t-1$ is broadly required for autophagy induction in many tissues upon heat stress. Since we previously showed that a hormetic HS increases the mRNA levels of autophagy-related genes $^{2}$, we performed quantitative RT-PCR analyses in sqst-1 (ok2892) mutants, and found that autophagy-related genes were increased in sqst-1(ok2892) mutants after HS similarly to WT animals (Supplementary Fig. 4a). sqst-1(ok2892) mutants similarly induced the heat-shock responsive gene $h s p-70$ compared with WT animals (Supplementary Fig. 4b). Since sqst-1(ok2892) mutants could induce heat-shock responsive- and autophagy genes following a hormetic HS, sqst-1 may function downstream of the transcriptional responses induced by HS. Altogether, our data suggest that HS-induced autophagy is sqst-1-dependent in the entire organism, whereas sqst-1 is required for basal autophagy specifically in neurons.

sqst-1 is required for the benefits of a hormetic heat shock. In addition to inducing autophagy, a hormetic HS early in life of C. elegans increases longevity, improves stress resistance, and is sufficient to prevent aggregation of intestinal and neuronal expanded PolyQ proteins ${ }^{2}$. Since we found sqst- 1 to be required for autophagy induction after a hormetic HS, we asked whether sqst- 1 could help mediate the benefits of a hormetic HS. Notably, sqst-1(ok2892) mutants did not benefit from a hormetic HS on day 1 of adulthood as measured by thermo-resistance (Fig. $2 \mathrm{a}$ and Supplementary Table 1), or longevity (Fig. 2b and Supplementary Table 2), demonstrating that sqst-1 is required for the positive effect of hormetic HS on thermo-resistance and longevity.

In contrast, sqst-1 was not required for the long lifespan of other conserved longevity paradigms that have been linked to autophagy ${ }^{3}$, including daf-2/insulin/IGF-1 receptor mutants, germline-less $g l p-1$ mutants, animals with reduced mTOR levels (let-363 RNAi), and animals with reduced levels of components of the mitochondrial electron transport chain (cyc-1 RNAi) (Supplementary Data 1). This indicates that sqst-1, unlike core autophagy genes ${ }^{3}$, is not broadly implicated in lifespan extension in C. elegans, but instead is required under specific, potentially proteotoxic conditions, such as a hormetic HS.

Finally, to determine whether selective autophagy mediated by sqst-1 could be involved in the removal of protein aggregates, we used C. elegans expressing tissue-specific YFP-tagged PolyQ proteins ${ }^{41-43}$. In C. elegans, PolyQ proteins accumulate with age $^{41}$, and have been shown to be ubiquitinated ${ }^{44}$. In contrast to the increased PolyQ aggregation observed upon reduction of core autophagy genes ${ }^{2,45}$, PolyQ aggregation was unchanged under basal conditions in neurons of sqst-1(ok2892) mutants (Fig. 2c). This result suggests that sqst-1-dependent autophagy is not required for basal degradation of neuronal PolyQ aggregates. In contrast to WT animals in which a hormetic HS reduces aggregation $^{2}$, sqst-1(ok2892) animals expressing neuronal PolyQ proteins showed an increased aggregate load in neurons following a hormetic HS (Fig. 2c). Moreover, fluorescence recovery after photobleaching (FRAP) experiments revealed that HS increased the mobility of the neuronal PolyQ aggregates in WT, but not in sqst-1(ok2892) mutant animals (Fig. 2d, e), while having only limited effects on diffuse neuronal PolyQ proteins (Supplementary Fig. 5). Consistently, a hormetic HS increased the soluble portion and decreased the insoluble portion of PolyQ proteins in WT, but not in sqst-1(ok2892) mutant animals (Supplementary Fig. 6). Similar to aggregate load (Fig. 2c), the baseline values for WT and sqst-1(ok2892) animals were not significantly different in FRAP (Fig. 2d, e) or in differential detergent extraction experiments (Supplementary Fig. 6). Furthermore, sqst-1(ok2892) mutants expressing neuronal PolyQ proteins were not significantly longer lived following a hormetic HS (Supplementary Table 2). Collectively, these assays show that sqst-1 is required for the benefits of a hormetic HS on neuronal proteostasis. In contrast, sqst-1 was only partially required for intestinal proteostasis in terms of PolyQ aggregation, while musclespecific PolyQ aggregation was unaffected by a hormetic HS or sqst-1(ok2892) mutation (Supplementary Fig. 7 and Supplementary Table 2, i.e., aggregate number, FRAP, and lifespan). Taken together, these data suggest that $s q s t-1$ is required when 
a

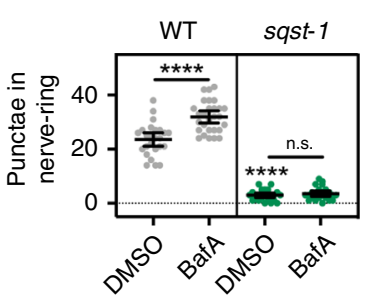

e
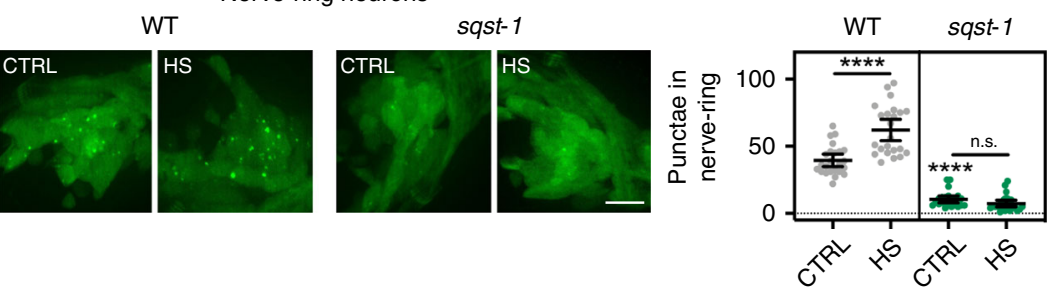

b

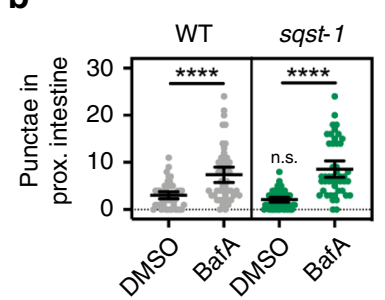

f
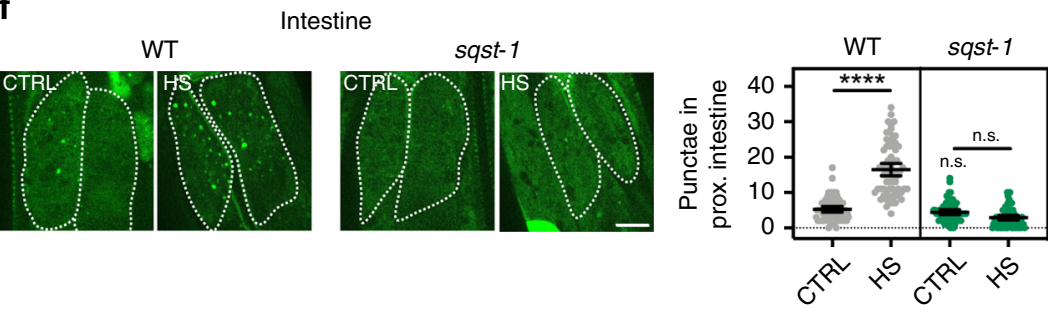

C

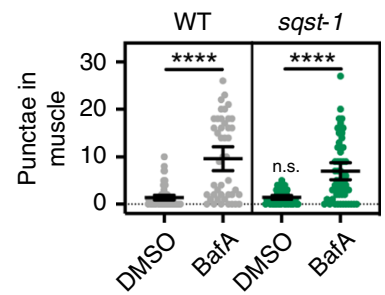

g
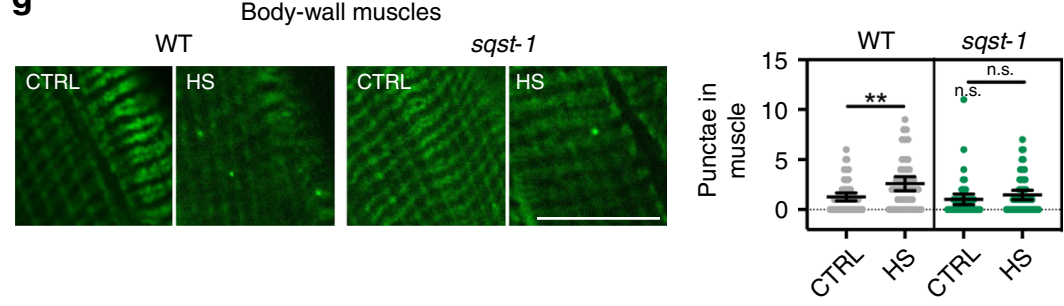

d

h

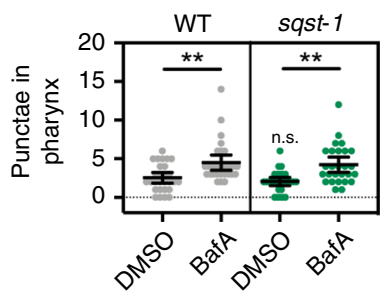

Pharynx
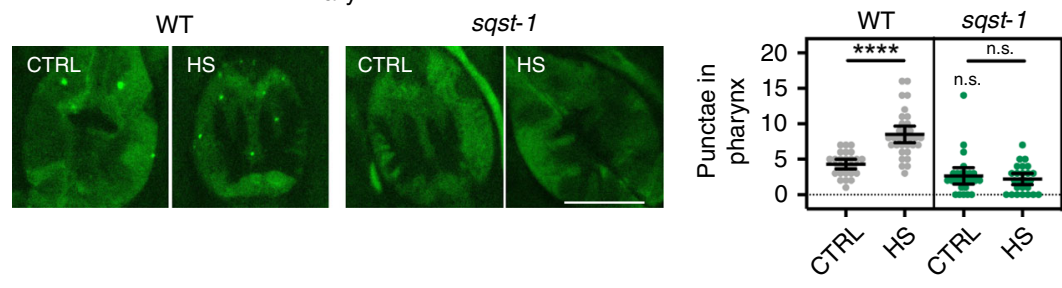

Fig. 1 sqst- 1 is required for autophagy induction after hormetic heat shock (HS). a-d Autophagy flux was measured at $20^{\circ} \mathrm{C}$ in WT and sqst-1(ok2892) (sqst-1) animals on day 1 of adulthood expressing rgef-1p::gfp::lgg-1 (a) or lgg-1p:::gfp::lgg-1 (b-d). WT and sqst-1 animals were injected with vehicle (DMSO) or bafilomycin A1 (BafA) to block autophagy at the lysosomal acidification step. GFP::LGG-1/Atg8-positive punctae were quantified from three independent experiments in a nerve-ring neurons (WT-DMSO, $N=25$; WT-BafA, $N=28$; sqst-1-DMSO, $N=25$, sqst-1-BafA, $N=24$ animals), b proximal intestinal cells (WT-DMSO, $N=49$; WT-BafA, $N=50$; sqst-1-DMSO, $N=55$, sqst-1-BafA, $N=46$ cells), c body-wall muscle (WT-DMSO, N=71; WT-BafA, N=44; sqst-1-DMSO, $N=48$, sqst-1-BafA, $N=53$ animals), and $\mathbf{d}$ and the terminal pharyngeal bulb (WT-DMSO, $N=28 ; \mathrm{WT}-\mathrm{BafA}, N=29 ;$ sqst-1-DMSO, $N=28$, sqst-1-BafA, $N=27$ animals), Error bars indicate $95 \% \mathrm{Cl}$. ns: $P>0.05,{ }^{\star \star} P<0.01,{ }^{\star \star \star \star} P<0.0001$, by two-way ANOVA with Tukey's multiple comparisons test. e-h GFP::LGG-1/Atg8-positive punctae were counted in WT and sqst-1(ok2892) (sqst-1) animals on day 1 of adulthood expressing rgef-1p::gfp::Igg-1 (e) or lgg-1p::gfp::Igg-1 (f-h). Animals were maintained under control conditions $15^{\circ} \mathrm{C}$ (CTRL) or subjected to HS for $1 \mathrm{~h}$ at $36^{\circ} \mathrm{C}$ (HS). Animals were imaged at time of highest induction in autophagy as previously characterized ${ }^{2}$. GFP::LGG-1/Atg8 punctae were quantified from three independent experiments in e nerve-ring neurons (WT-CTRL, $N=24$; WT-HS, $N=23$; sqst-1-CTRL, $N=23$, sqst-1-HS, $N=24$ animals) with $2 \mathrm{~h}$ of recovery after HS, $\mathbf{f}$ proximal intestinal cells (WT-CTRL, $N=67$; WT-HS, $N=70$; sqst-1-CTRL, $N=66$, sqst-1-HS, $N=71$ cells) with $4 \mathrm{~h}$ of recovery after HS, $\mathbf{g}$ body-wall muscle (WTCTRL, $N=60$; WT-HS, $N=48$; sqst-1-CTRL, $N=50$, sqst-1-HS, $N=60$ animals) (no recovery), and $\mathbf{h}$ terminal pharyngeal bulb (WT-CTRL, $N=26$; WT$\mathrm{HS}, \mathrm{N}=32$; sqst-7-CTRL, $N=26$, sqst-1-HS, $N=25$ animals) with $4 \mathrm{~h}$ of recovery after HS. Error bars indicate $95 \% \mathrm{Cl}$. ns: $P>0.05,{ }^{\star \star} P<0.01,{ }^{\star \star \star \star} P<$ 0.0001 , by two-way ANOVA with Tukey's multiple comparisons test. Scale bar: $10 \mu \mathrm{m}$. Source data are provided in the Source Data file.

autophagy is induced by a hormetic HS and plays a key role in improving proteostasis particularly in neurons of PolyQexpressing animals.

Overexpression of SQST-1 is sufficient to extend lifespan. We previously showed that a hormetic HS leads to an increase in the transcription of $s q s t-1^{2}$, and observed that sqst-1 mRNA levels remained upregulated for several days after a HS (Supplementary Fig. 4c). Given the requirement of sqst-1 for HS-induced autophagy (Fig. 1) and the organismal benefits of a hormetic HS (Fig. 2), we next tested whether increasing sqst-1 levels in C. elegans was sufficient to induce organismal benefits, similar to a hormetic HS. To this end, we overexpressed sqst-1 fused to $g f p$ under the control of its endogenous promoter and found SQST-1::GFP to be expressed mainly in punctate structures in head neurons and in the pharynx throughout adulthood (Supplementary Fig. 8a). SQST-1::GFP accumulated upon autophagy block (Supplementary Fig. 8b), as observed for untagged SQST $-1{ }^{31}$, indicating that the SQST-1::GFP protein is 
a

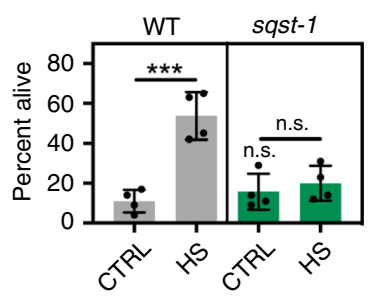

b

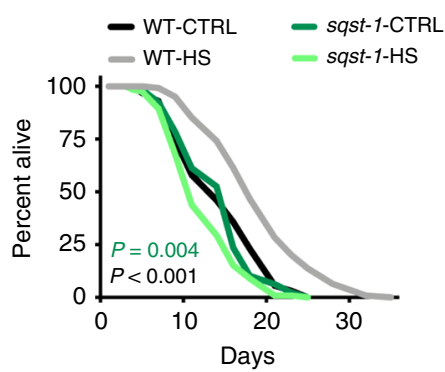

C

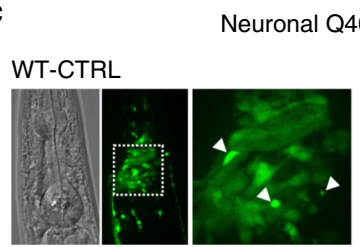
sqst-1-CTRL
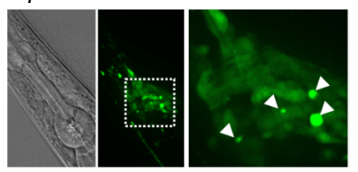
WT-HS

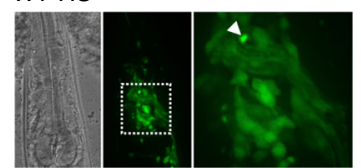

sqst-1-HS
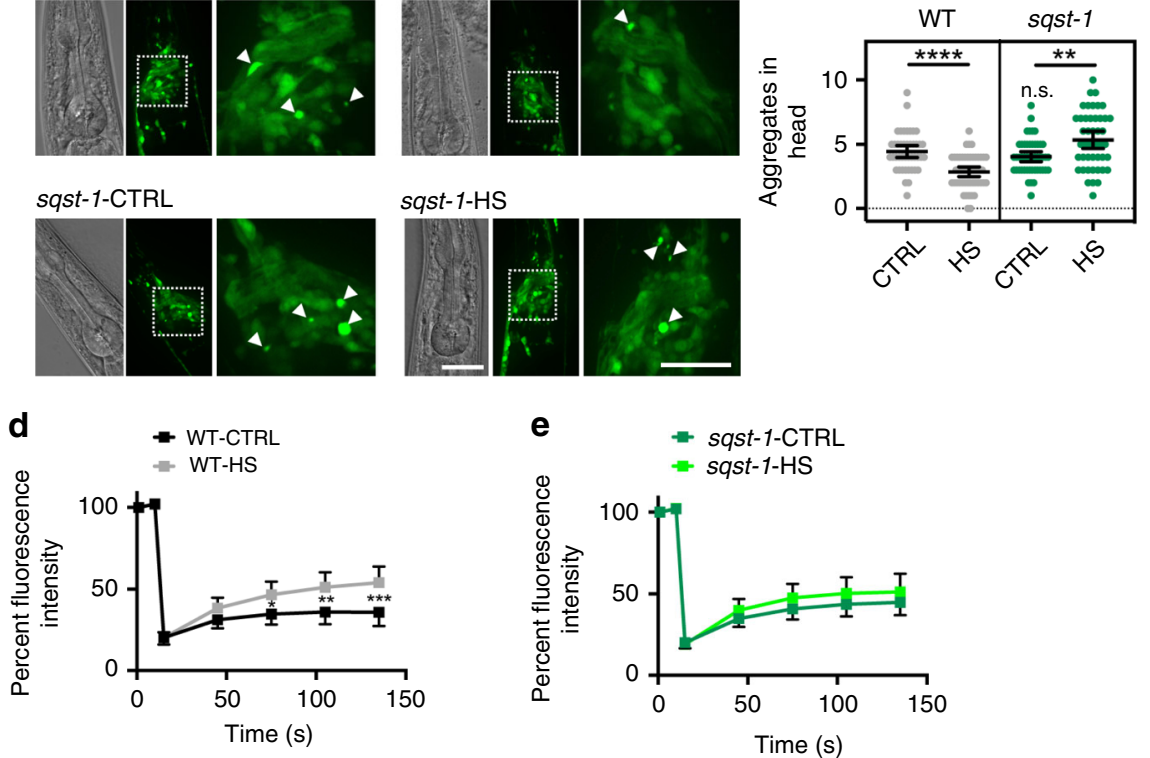

Fig. 2 sqst -1 is required for the organismal benefits of a hormetic heat shock (HS). a Survival of WT (N2) and sqst-1(ok2892) (sqst-1) animals subjected to $\mathrm{HS}$ on day 1 of adulthood, and then incubated for $7 \mathrm{~h}$ at $36^{\circ} \mathrm{C}$ on day 3 of adulthood ( $n=4$ plates). Error bars indicate SD, ns: $P>0.8, \star \star \star P<0.001$ by twoway ANOVA with Tukey's multiple comparisons test. See Supplementary Table 1 for experimental details and additional repeats. $\mathbf{b}$ Lifespan analysis of WT and sqst-1(ok2892) (sqst-1) animals at $20^{\circ} \mathrm{C}$ subjected to $1 \mathrm{~h} \mathrm{HS}$ at $36^{\circ} \mathrm{C}$ on day 1 of adulthood. WT-CTRL animals $(N=126)$ compared with WT-HS animals $(N=110): P<0.0001$; sqst-1-CTRL animals $(N=128)(P=0.3$ to WT) compared with sqst-1-HS animals $(N=115): P=0.004$, by log-rank test. See Supplementary Table 2 for experimental details and additional repeats. c Neuronal PolyQ (rgef-1p::Q40::yfp) aggregates detected on day 4 of adulthood in WT and sqst-1(ok2892) (sqst-1) animals maintained under control conditions ( $20^{\circ} \mathrm{C}, \mathrm{CTRL}$ ) or subjected to $\mathrm{HS}\left(1 \mathrm{~h}\right.$ at $\left.36^{\circ} \mathrm{C}\right)$ on day 1 of adulthood (HS). Scale bar $20 \mu \mathrm{m}$. The number of neuronal PolyQ aggregates were quantified from three independent experiments in $\mathrm{WT}-\mathrm{CTRL}, \mathrm{N}=43 ; \mathrm{WT}-\mathrm{HS}, \mathrm{N}=50$; sqst-1-CTRL, $N=48$, sqst-1-HS, $N=45$ animals. Error bars indicate $95 \% \mathrm{Cl}$. ns: $P>0.6,{ }^{\star \star} P<0.01,{ }^{\star \star \star \star} P<0.0001$, by two-way ANOVA with Tukey's multiple comparisons test. Scale bar 20 and $10 \mu \mathrm{m}$ for close-up. d, e FRAP measurements of neuronal PolyQ (rgef-1p::Q40::yfp) aggregates on day 8 of adulthood in WT (e) and sqst-1(ok2892) (sqst-1) (f) animals maintained under control conditions $\left(20^{\circ} \mathrm{C}, \mathrm{CTRL}\right)$ or subjected to $\mathrm{HS}\left(1 \mathrm{~h}\right.$ at $\left.36^{\circ} \mathrm{C}\right)$ on day 1 of adulthood (HS). The fluorescence signal was bleached after $20 \mathrm{~s}$ and fluorescence recovery was measured for 2 min. The average $\%$ of fluorescence intensity was quantified from three independent experiments in WT-CTRL, $N=32 ; \mathrm{WT}-\mathrm{HS}, \mathrm{N}=30 ;$ sqst-1-CTRL, N=32, sqst-1-HS, N=31 animals. Error bars indicate $95 \% \mathrm{Cl} .{ }^{\star \star} P<0.05,{ }^{\star \star} P<0.01,{ }^{\star \star \star} P<0.001$, by two-way ANOVA with Sidak's multiple comparisons test. $P>0.05$ for all timepoints when comparing WT-CTRL and sqst-1-CTRL by two-way ANOVA with Sidak's multiple comparisons test. Source data are provided in the Source Data file.

functional as it can serve as a substrate for autophagy. Animals overexpressing SQST-1 (GFP-tagged or untagged), either from extrachromosomal arrays or from integrated loci, displayed an average lifespan extension of $\sim 30 \%$ (Fig. 3a and Supplementary Data 2). Integrated SQST-1::GFP-expressing animals had a 30-100-fold increase in SQST-1 levels, whereas animals overexpressing untagged SQST-1 from extrachromosomal arrays in the sqst-1(syb764) mutant background had a $\sim 2-7$-fold increase in SQST-1 levels (Supplementary Fig. 8c, d), highlighting that a range of SQST-1 overexpression resulted in lifespan extension. Importantly, in the sqst-1(syb764) mutant, overexpression of an untagged, truncated SQST-1 protein without the UBA domain
(SQST-1 $\triangle \mathrm{UBA}$ ), which enables p62 to bind ubiquitinated proteins ${ }^{4}$, did not extend lifespan (Fig. 3b, Supplementary Data 2; Supplementary Fig. 8d). These observations indicate that the interaction of SQST-1 with ubiquitinated proteins upon SQST-1 overexpression is required for the lifespan-extending effects of SQST-1 overexpression.

Given that sqst-1 was required for autophagosome formation in neurons (Fig. 1a, e), and because the SQST-1::GFP expression was primarily observed in neurons surrounding the pharynx (Supplementary Fig. 8a), we overexpressed SQST-1::GFP under the control of a pan-neuronal promoter ( $r g e f-1 p)$. Transgenic animals overexpressing neuronal SQST-1::GFP (>100-fold increase in 
a
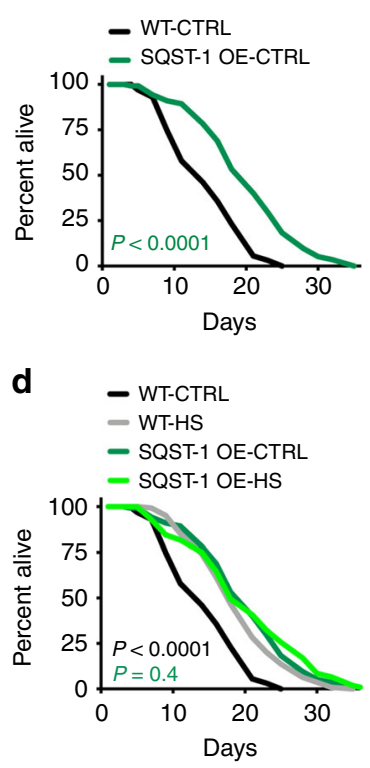

f

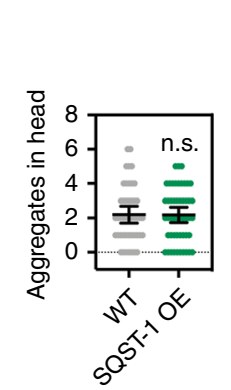

g b

- WT

- sqst-1

- sqst-1:SQST-1

- sqst-1; SQST-1 $\triangle \mathrm{UBA}$

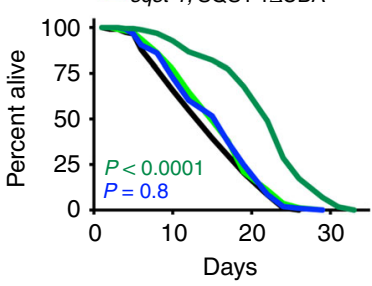

e

e $\quad$ - WT-CTRL

- WT-HS

- Neuronal SQST-1 OE-CTRL

- Neuronal SQST-1 OE-HS

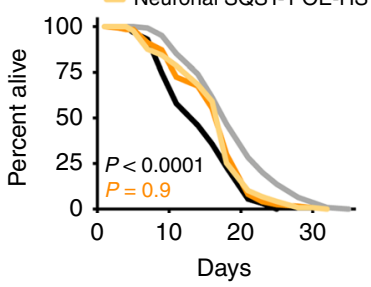

FRAP of neuronal Q40

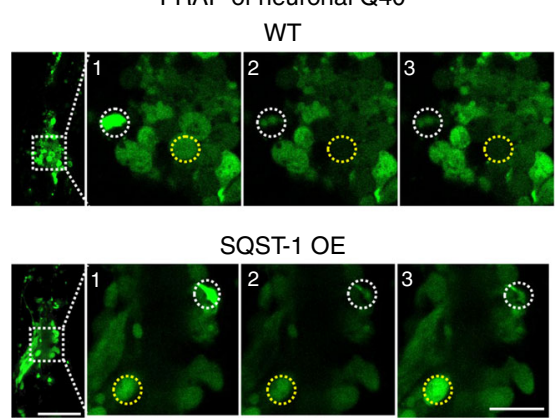

C

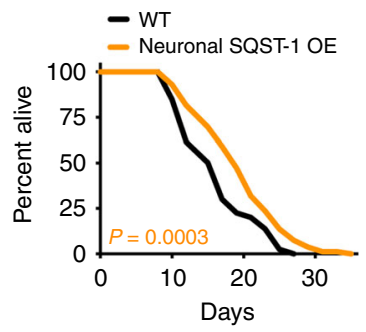

h

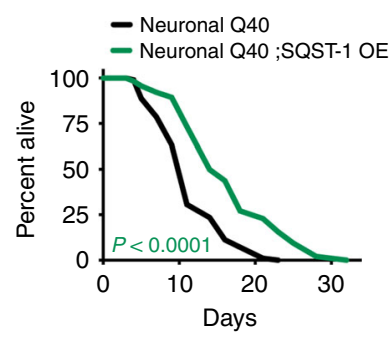

Neuronal Q40 aggregates

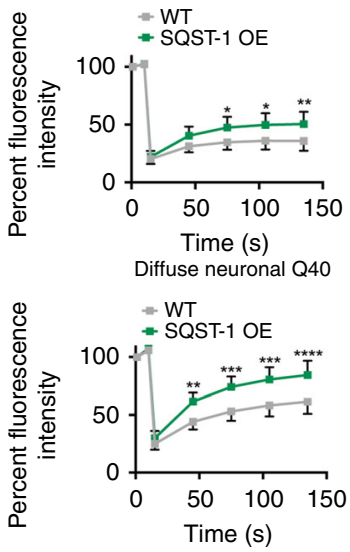

Fig. 3 SQST-1 overexpression extends C. elegans lifespan and improves proteostasis. a Lifespan analysis of WT (N2) (N=58 animals) and SQST-1 OE (sqst-1p:::sqst-1::gfp (1)) ( $N=88$ animals) animals at $20^{\circ} \mathrm{C}: P$-value by log-rank test. b Lifespan analysis of WT (N2) ( $N=92$ animals), sqst-1(syb764) ( $N=$ 88 animals), and sqst-1(syb764) animals expressing either sqst-1p:::sqst-1 + rol-6 (sqst-1; SQST-1) ( $N=124$ animals) or sqst-1p::sqst-1 $1 \Delta U B A+$ rol-6 (sqst-1; SQST-1 $\triangle$ UBA) $\left(N=107\right.$ animals) at $20^{\circ} \mathrm{C}$. P-values by log-rank test. c Lifespan analysis of WT (N2) (N=85 animals) and neuronal SQST-1 OE (rgef-1p:: sqst-1::gfp (1)) ( $N=85$ animals) animals at $20^{\circ} \mathrm{C}$. P-value by log-rank test. d Lifespan analysis of WT (N2) and SQST-1 OE (sqst-1p:::sqst-1::gfp (1)) animals subjected to hormetic HS on day 1 of adulthood. WT-CTRL, $N=126$; WT-HS, $N=111$; SQST-1 OE-CTRL, $N=116, S Q S T-1$ OE-HS, $N=106$ animals at $20{ }^{\circ} \mathrm{C}$. P-values by log-rank test. e Lifespan analysis of WT (N2) and neuronal SQST-1 OE (rgef-1p::sqst-1::gfp (1)) animals subjected to hormetic HS on day 1 of adulthood. WT-CTRL, $N=126$; WT-HS, $N=111$; Neuronal SQST-1 OE-CTRL, $N=123$, SQST-1 OE-HS, $N=120$ animals at $20^{\circ} \mathrm{C}$. $P$-values by log-rank test. a-e See Supplementary Data 2 and Table 3 for experimental repeats. $\mathbf{f}$ Quantification of neuronal PolyQ aggregates (rgef-1p::Q40::yfp) on day 5 of adulthood from three independent experiments in WT $(N=31$ animals) and SQST-1 OE animals (sqst-1p::sqst-1 + rol-6) $(N=30$ animals). Error bars indicate $95 \% \mathrm{Cl}$. ns: $P>0.05$ by $t$-test. $\mathbf{g}$ Fluorescence images and FRAP measurements of the aggregated (white dashed circles) and diffuse (yellow dashed circles) portion of neuronal PolyQ (rgef-1p::Q40::yfp) on day 8 of adulthood in WT and SQST-1-overexpressing animals sqst-1p::sqst-1+rol-6 (SQST-1 OE) at $20^{\circ} \mathrm{C}$. Fluorescence signal bleached after $10 \mathrm{~s}$ (timepoint 2) and fluorescence recovery was measured for 2 min. The average \% of fluorescence intensity was quantified from three independent experiments in WT, $N=32$; SQST-1 OE, $N=31$. Image numbers correspond to the indicated timepoints. Error bars indicate $95 \% \mathrm{Cl}$. All measurements are $P>0.05$ by two-way ANOVA with Sidak's multiple comparisons test unless indicated otherwise ${ }^{\star} P<0.05,{ }^{\star \star} P<0.01,{ }^{\star \star \star} P<0.001,{ }^{\star \star \star \star} P<0.0001$. Outline of pharyngeal bulbs in image of head region. Scale bar: 20 and $10 \mu \mathrm{m}$ for close-up. h Lifespan analysis of Neuronal Q40-expressing animals (rgef-1p::Q40::yfp) ( $N=39$ animals) and Neuronal Q40; SQST-1 OE (rgef-1p::Q40::yfp; sqst-1p:: sqst- $1+$ rol-6) $(\mathrm{N}=79$ animals $)$ at $20^{\circ} \mathrm{C}$. P-value by log-rank test. See Supplementary Table 4 for experimental repeats. Source data are provided in the Source Data file. 
integrated lines) displayed up to $\sim 25 \%$ lifespan extension at $20^{\circ} \mathrm{C}$, albeit with some variability and most consistently when SQST-1 was expressed from extrachromosomal arrays (Fig. 3c, Supplementary Data 2 and Supplementary Table 3). Of note, our collection of SQST-1::GFP-overexpressing strains generally displayed healthspan parameters similar to WT animals (Supplementary Fig. 9). Collectively, these data show that overexpression of a selective autophagy receptor, SQST-1, is sufficient to extend C. elegans lifespan.

Since we found that sqst-1 was required for the lifespan increase mediated by a hormetic HS (Fig. 2d), we tested whether subjecting SQST-1-overexpressing animals to a hormetic HS would be additive for lifespan. The lifespan of animals overexpressing SQST-1::GFP under the endogenous promoter or under the neuronal rgef-1 promoter was unchanged after a hormetic HS (Fig. 3d, e and Supplementary Table 3). These results, together with the requirement of $s q s t-1$ for the benefits of the hormetic HS (Fig. 2), are consistent with hormetic HS and SQST-1 overexpression extending lifespan by common mechanisms.

SQST-1 overexpression improves proteostasis. Since sqst-1 was required for hormetic HS to alleviate neuronal PolyQ aggregation (Fig. 2c), we next investigated whether SQST-1 overexpression could similarly alleviate neuronal PolyQ aggregation. While the number of neuronal PolyQ aggregates in SQST-1-overexpressing animals was unchanged (Fig. 3f), the mobility of both the aggregated as well as the diffuse PolyQ proteins was increased upon overexpression of SQST-1 (Fig. 3g). These data suggest that SQST-1 overexpression changes the properties of aggregationprone PolyQ::YFP. Furthermore, SQST-1 overexpression was sufficient to extend the lifespan of animals expressing neuronal PolyQ proteins (Fig. 3h), as well as animals carrying temperaturesensitive (ts) missense mutations in genes causing protein misfolding and tissue-specific protein aggregation ${ }^{46,47}$, i.e., dynamin GTPase (dyn-1(ts); neurons), Ras (let-60(ts); intestine), and myosin (unc-54(ts); muscle) (Supplementary Table 4). Taken together, SQST-1 overexpression was able to improve proteostasis and longevity in $C$. elegans with protein-folding defects.

p62 can mediate the degradation of aggregation-prone proteins $^{8-12}$. Therefore, we next asked by which mechanisms SQST-1 overexpression mediated proteostasis in C. elegans. We found that both endogenous and neuronal overexpression of SQST-1::GFP reduced the levels of ubiquitinated proteins compared with WT animals (Supplementary Fig. 10a). Since the degradation of ubiquitinated proteins can occur via the ubiquitinproteasome system (UPS) as well as autophagic degradation, we next tested whether SQST-1-overexpressing animals exhibited elevated proteasome activity using a fluorogenic peptide substrate specific for the chymotrypsin-like activity of the proteasome ${ }^{48}$. We found that $g l p-1$ mutants, previously reported to exhibit increased proteasomal activity ${ }^{48}$, had an increase of $100 \%$ in their proteasome activity, whereas animals overexpressing SQST-1 under the endogenous promoter (SQST-1-overexpressing line 1 and 2 ) had either a $\sim 10 \%$ marginal or $\sim 30 \%$ significant increase, respectively; and animals overexpressing SQST-1 in the neurons alone had a modest $\sim 15 \%$ increase (Supplementary Fig. 10b). Since the proteasomal activity of SQST-1-overexpressing animals was only modestly increased, we next examined whether an induction of autophagy could contribute to the increased organismal health and proteostasis upon SQST-1 overexpression. First, we investigated if autophagy was required for the lifespan extension observed in SQST-1-overexpressing animals. We fed SQST-1-overexpressing animals bacteria expressing dsRNA targeting distinct autophagy-related genes from adulthood on.
We found that RNAi targeting autophagy genes involved in different steps of the autophagy process, such as $\lg g-1 / A T G 8$ (Fig. 4a), which is part of the Atg8-conjugation system, or cup-5 (Fig. 4b), whose loss impairs degradation of autolysosomal contents ${ }^{49}$, abrogated the lifespan extension of SQST-1-overexpressing animals. A similar requirement was found for vps-34 and bec-1/Atg6, which are involved in nucleation of autophagosomal membranes, atg-18, which is involved in phagophore formation, and epg-5, which is involved in lysosomal degradation ${ }^{50}$ (Supplementary Data 3). Since multiple genes with functions in different steps of the autophagy process were similarly required for the lifespan extension conferred by overexpression of SQST-1, autophagy as a general process is likely required for the lifespan extension of SQST-1-overexpressing animals.

To explore whether autophagy was required for the effects of SQST-1-overexpression on proteostasis, we again used $d y n-1$ (ts),
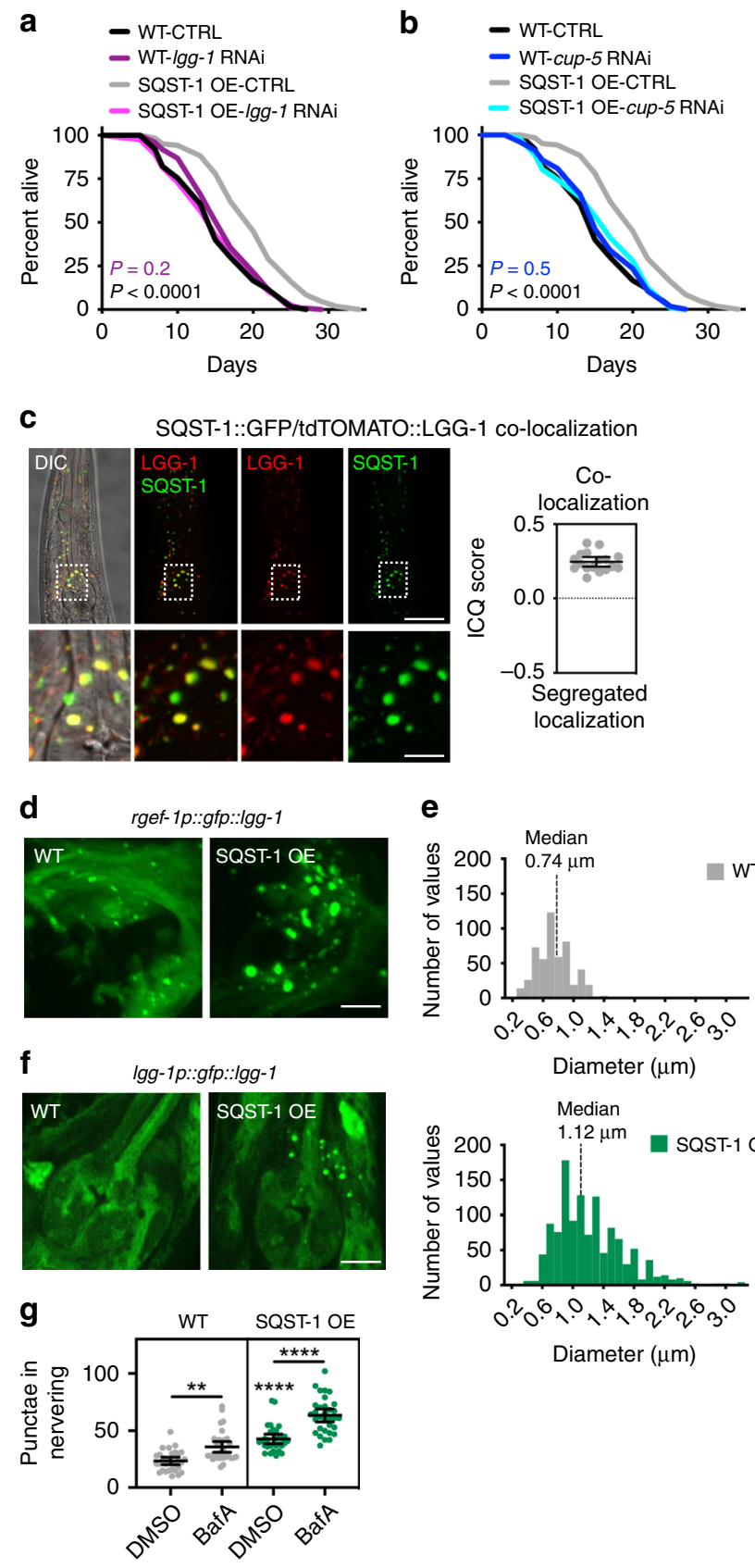
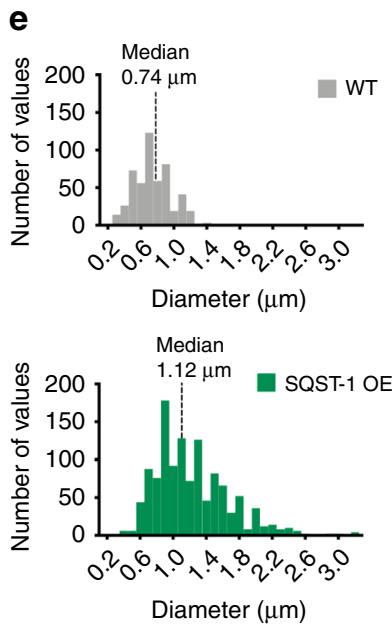
Fig. 4 SQST-1 overexpression induces neuronal autophagy. a, b Lifespan analysis of WT and sqst-1p:::sqst-1::gfp (SQST-1 OE) animals at $20^{\circ} \mathrm{C}$ subjected to bacteria expressing empty vector (CTRL) or dsRNA targeting autophagy genes (a) lgg-1/ATG8 and (b) cup-5 from day 1 of adulthood. WT-CTRL, $N=121$; WT-lgg-1/ATG8 RNAi, $N=121 ; \mathrm{WT}$-cup-5 RNAi, $N=124$; SQST-1 OE-CTRL, $N=108$, SQST-1 OE-lgg-1/ATG8 RNAi, $N=116$; SQST-1 OE-cup-5 RNAi, $N=129$ animals. $P$-values by log-rank test. See Supplementary Data 3 for experimental repeats. c Colocalization of SQST-1 and LGG-1/Atg8 in the head region of animals expressing sqst-1p:::sqst-1::gfp and lgg-1p::tdtomato::Igg-1. First panel is overlay of DIC (differential interference contrast) image and GFP (SQST-1) and tdTOMATO (LGG-1/Atg8) fluorescence. Second panel is overlay of GFP (SQST-1) and tdTOMATO (LGG-1/Atg8) fluorescence, followed by single fluorescent channels. Scale bar: 10 and $2.5 \mu \mathrm{m}$ in close-up. Intensity correlation quotient (ICQ) values between 0 and 0.5 indicate colocalization and values between 0 and -0.5 indicate segregated fluorescence. ICQ score were quantified in entire head region from two independent experiments ( $N=17$ animals). Error bars indicate $95 \% \mathrm{Cl}$. d GFP::LGG-1/Atg8-positive punctae in nerve-ring neurons of WT and SQST-1 OE (sqst-1p::sqst-1) animals on day 1 of adulthood expressing neuronal GFP::LGG-1 (rgef-1p::gfp::Igg-1). Scale bar: $10 \mu \mathrm{m}$. e Histogram of GFP::LGG-1/Atg8 structures binned by their diameter in nerve-ring neurons of WT ( $N=519$ GFP::LGG-1/Atg8 structures) and SQST-1 OE ( $N=1196$ GFP::LGG-1/Atg8 structures) animals with indicated median diameter. $P<0.0001$ by Wilcoxon signed rank test. See Supplementary Table 5. f GFP::LGG-1/Atg8-positive punctae in nerve-ring neurons of WT and SQST-1 OE (sqst-1p::sqst-1) animals on day 1 of adulthood expressing GFP::LGG-1 under its endogenous promoter (lgg-1p:::gfp::Igg-1). Scale bar: $10 \mu \mathrm{m}$. $\mathbf{g}$ Autophagy-flux measurements were performed at $20^{\circ} \mathrm{C}$ in WT and SQST-1 OE animals on day 1 of adulthood expressing rgef-1p:::gfp::lgg-1. WT and SQST-1 OE animals were injected with vehicle (DMSO) or bafilomycin A1 (BafA) to block autophagy at the lysosomal acidification step. GFP::LGG-1/Atg8-positive punctae were quantified from three independent experiments (WT-DMSO, $N=30$; WT-BafA, $N=32$; SQST-1 OE-DMSO, $N=31$, SQST-1 OE-BafA, $N=32$ animals). Error bars indicate $95 \% \mathrm{Cl}$. ${ }^{\star \star} P<0.01,{ }^{\star \star \star \star} P<0.0001$, by two-way ANOVA with Tukey's multiple comparisons test. Source data for $(\mathbf{a}),(\mathbf{b}),(\mathbf{c}),(\mathbf{e})$, and $(\mathbf{g})$ are provided in the Source Data file.

let-60(ts), and unc-54(ts) protein-folding mutants, since these strains allowed for co-expression of GFP-tagged SQST-1. Overexpression of SQST-1::GFP improved the movement defects of dyn-1(ts) and let-60(ts), but not muscle-specific unc-54(ts) mutants at the restrictive temperature in an autophagydependent manner (Supplementary Fig. 11a-c). Furthermore, SQST-1::GFP accumulated upon $\lg g$-1/ATG8 RNAi in nerve-ring neurons and distal intestines of $d y n-1(t s)$ and let-60(ts) mutants, but not in body-wall muscle of unc-54(ts) mutants, which under control conditions showed a striking increase in SQST-1::GFP accumulation (Supplementary Fig. 11d-f). These data indicate that the effects of SQST-1 on proteostasis are tissue-specific and that SQST-1 overexpression may improve proteostasis in neurons and intestine at least in part by engaging autophagy. Moreover, we found that overexpression of SQST-1::GFP in atg-18( $g k 378)$ hypomorphs, which display a high percentage of larval lethality ${ }^{51}$, significantly increased the survival of atg-18(gk378) larvae, while overexpressing LGG-1/Atg8 did not (Supplementary Fig. 12a, b). This indicates that SQST-1-overexpressing animals could at least partially overcome the autophagy defects caused by the hypomorphic mutation in atg-18/WIPI. Taken together, our data support the notion that SQST-1 overexpression in C. elegans improves neuronal and intestinal proteostasis upon proteotoxic conditions in an autophagy-dependent manner, possibly by facilitating the elimination of protein aggregates.
Overexpression of SQST-1 induces neuronal autophagy. Since autophagy-related genes were required for the SQST-1mediated benefits on lifespan and proteostasis (Fig. 3), we explored whether SQST-1 overexpression was sufficient to regulate autophagy. First, we asked whether SQST-1 colocalized with LGG-1/Atg8 in adult C. elegans co-expressing

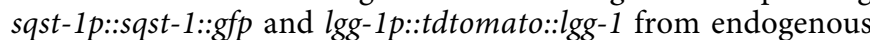
promoters. Most of the SQST-1::GFP structures observed in and around the nerve ring of day 1 adult animals co-localized with tdTOMATO::LGG-1 (Fig. 4c), consistent with SQST-1 interacting with LGG-1/Atg8 in adult C. elegans, as previously shown in embryos ${ }^{52}$.

We next monitored autophagy in individual tissues of C. elegans expressing GFP-tagged LGG-1/Atg8 and overexpressing untagged SQST-1. Endogenous SQST-1 overexpression led to a marked increase in the number as well as size of GFP::LGG-1/Atg8-positive structures around the nerve ring compared with WT (Fig. 4d, e, Supplementary Fig. 12c), whereas a lipidation-deficient GFP::LGG-1(G116A) reporter showed few 'background' punctae, irrespective of SQST-1 levels (Supplementary Fig. 12c). Notably, the median diameter of neuronal GFP::LGG-1/Atg8-positive punctae was $\sim 400 \mathrm{~nm}$ larger in SQST-1-overexpressing animals compared with WT animals (Fig. 4e and Supplementary Table 5), suggesting that SQST-1 overexpression potentiates an enlargement of the autophagosome in neurons.

Under basal conditions, the endogenously expressed GFP::LGG-1/Atg8 reporter does not allow for a clear separation of neurons from other tissues in the head region ${ }^{53}$. In SQST-1-overexpressing animals, however, enlarged GFP::LGG-1/Atg8-positive punctae were observed in the region of the nerve-ring neurons, which provides further evidence for the increased formation of enlarged autophagic structures in that tissue (Fig. 4f). We also observed an increase in the number and size of GFP::LGG-1/Atg8-positive puncta in the intestine and the pharynx of these animals (Supplementary Fig. 12d-g and Supplementary Table 5).

To further assess the dynamic status of the autophagy process, we injected SQST-1-overexpressing animals with BafA in autophagy-flux assays. The number of GFP::LGG-1/Atg8-positive punctae in neurons and the intestine of both WT and SQST-1overexpressing animals increased upon BafA injection (Fig. 4h and Supplementary Fig. 12h), consistent with an induction of autophagy in these tissues. In contrast, the pharynx of SQST-1overexpressing animals showed no change in the number of GFP::LGG-1/Atg8-positive punctae after BafA injection (Supplementary Fig. 12i), indicating a block in autophagy in this tissue. Thus, our results indicate that SQST-1 overexpression leads to autophagy induction in neurons and the intestine, which supports the idea that SQST-1-mediated longevity and improved proteostasis upon proteotoxic conditions occurs at least in part by the induction of tissue-specific autophagy.

\section{Discussion}

While autophagy as a process has been linked to aging and age-related diseases, the role of selective autophagy remains less clear. Here we demonstrate an important role for the selective autophagy receptor SQST-1 in aging and proteostasis, since we found sqst-1 to be required for the organismal benefits of a hormetic HS in C. elegans, and overexpression of SQST-1 was sufficient to extend lifespan and improve proteostasis in an autophagy-dependent manner. Collectively, our studies indicate that SQST-1, and possibly selective autophagy, plays an important role in longevity and health upon proteotoxic conditions. 
We have previously shown that core autophagy genes, including $\operatorname{lgg}-1 / A T G 8$ and $b e c-1 / A T G 6$, are required for the lifespan extension induced by hormetic HS $^{2}$ as well as by multiple conserved longevity paradigms ${ }^{3}$. In contrast, we found that sqst-1 was not required for the longevity of long-lived daf-2 insulin/IGF1 receptor mutants, germline-less $g l p-1$ mutants, and animals with reduced mTOR levels (let-363 RNAi) or mitochondrial respiration (cyc-1 RNAi), indicating a context-specific requirement for $s q s t-1$ in lifespan. Since sqst-1 is required for lifespan extension caused by reduced levels of the mitochondrial protein frataxin $f r h-1^{30}$, which leads to iron depletion and the induction of stress responses ${ }^{23,54}$, we propose that $s q s t-1$ may be specifically required in mediating selective autophagy under conditions of proteotoxic stress.

While sqst- 1 was required systemically for autophagy induction in response to a hormetic HS, loss of sqst-1 did not abrogate PolyQ aggregation under basal conditions, which is in contrast to the loss of core autophagy genes that has been shown to increase PolyQ aggregates 2,45 . This may indicate that PolyQ aggregates in C. elegans are either turned over by bulk autophagy rather than sqst-1-mediated aggrephagy, or that bulk autophagy plays a role in preventing the formation of PolyQ aggregates possibly by the degradation of soluble PolyQ. Under basal conditions, sqst-1 was instead specifically required for autophagosome formation in neurons. This implies that autophagy in non-neuronal tissues is mediated in an sqst-1-independent manner, and suggests that sqst-1-mediated neuronal autophagy may not be important for lifespan determination under non-stress conditions in C. elegans. Since sqst-1 mutants had similar levels of PolyQ aggregation in neurons compared with WT animals, it is possible that there are sqst-1-dependent and independent autophagy pathways in $C$. elegans neurons. Such a possible sqst-1-independent autophagy pathway must, however, be $\operatorname{lgg}$-1/Atg8-independent, since sqst-1 mutants were essentially void of GFP::LGG-1-positive punctae. To this point, it would be of interest to investigate neuronal roles for LGG-2, another Atg8 homolog in C. elegans, in future experiments.

While sqst-1 was only required upon proteotoxic conditions, we show here that endogenous overexpression as well as neuronal overexpression of an autophagy receptor, SQST-1, led to longevity at $20^{\circ} \mathrm{C}$. Furthermore, SQST-1 overexpression was sufficient to increase the mobility of neuronally expressed PolyQ proteins. This indicates that increased SQST-1 levels can change the nature of PolyQ proteins toward increased mobility, and thus possibly improved degradation. It will be interesting to determine how SQST-1 affects the biophysical properties of PolyQ proteins. One possibility could be that increased SQST-1 association with PolyQ aggregates modulates the nature of the aggregates, making them cytoprotective rather than detrimental for the cell as previously suggested $^{9}$. Alternatively, it is possible that SQST-1 overexpression increases the degradation of PolyQ proteins, which could thus decrease the overall concentration and consequently increase the mobility of PolyQ proteins. Future experiments are needed to address these possibilities.

We found that SQST-1-overexpressing animals have reduced levels of ubiquitinated proteins, which could be due to increased degradation of ubiquitinated proteins, or due to changes in the abundance or activity of ubiquitin ligases or deubiquitinating enzymes. While we have not addressed the latter possibility, we found that SQST-1 overexpressing animals have increased proteasome activity, albeit modestly compared with germline-less $g l p-1$ mutants $^{48}$. This, in combination with the increased autophagy flux in SQST-1-overexpressing animals, supports the idea that increasing SQST-1 levels increases protein degradation capabilities in C. elegans. Such increased protein degradation capabilities could contribute to the proteostasis and lifespan- extending benefits observed in these animals. Importantly, we demonstrate here that SQST-1 overexpression can improve proteostasis in a $C$. elegans neurodegenerative-disease model. p62 overexpression has been shown to alleviate symptoms in an Alzheimer's disease mouse model by the induction of autophagy ${ }^{10}$. We show that the improved proteostasis of animals with temperature-sensitive missense aggregation mutations similarly required autophagy genes, suggesting that autophagy, at least in part, is an important effector mechanism of the beneficial effects of SQST-1 overexpression.

Consistently, we showed that endogenous SQST-1 overexpression can lead to the induction of autophagy in neurons and the intestine. This leads to the question of how SQST-1 could mediate autophagosome induction. Recent findings point to the intriguing possibility that the presence of cargo can induce the formation of the autophagic isolation membranes ${ }^{55}$. This is supported by findings that p62 is present at the autophagosomeformation site before the interaction with LC3/Atg8 in mouseembryonic fibroblasts ${ }^{6,56}$. In addition, ubiquitinated cargo may directly contribute to the recruitment of the autophagy machinery ${ }^{57}$, and induce p62 phase separation and cargo segregation in vitro ${ }^{58}$. Thus, it seems plausible that ubiquitinated proteins that accumulate upon a hormetic HS in C. elegans, induce autophagy via recruitment of SQST-1. This suggests the possibility, that $C$. elegans neurons are prone to accumulate increased levels of ubiquitinated proteins under basal conditions that could be cleared more efficiently upon SQST-1 overexpression. Alternatively, increased SQST-1 levels, particularly in neurons, could lead to the formation of ubiquitinated aggregates, which are somehow "primed" for removal by autophagy, causing organismal benefits.

Since SQST-1 overexpression led to the formation of unusually large autophagosomes in neurons, it is similarly interesting to speculate on how SQST-1 could mediate autophagosome size. Studies in yeast have shown that the amount of Atg8 can regulate the size of autophagosomes by controlling the expansion of the phagophore ${ }^{59}$. Recent in vitro reconstitution models suggest that p62 oligomerization into filaments enables the formation of large clusters with ubiquitinated proteins that at the same time can interact with the isolation membrane ${ }^{55,60}$. This interaction facilitates the bending of the membrane around the cargo ${ }^{55,60}$. Therefore, it is conceivable that increased SQST-1 protein levels could drive the formation of larger filaments and thus dictate the size of autophagosomes. It is possible that such larger autophagosomes are able to degrade ubiquitinated proteins, since SQST-1 overexpression reduced the steady-state levels of ubiquitinated proteins in C. elegans. Recent evidence suggests that the degradation of specific cargos by selective autophagy could be important for maintaining health ${ }^{3}$. It would therefore be interesting to further explore what the content of these larger autophagic structures is and their specific role in individual tissues, including in the pharynx in which SQST-1 overexpression caused a block in autophagy.

In conclusion, this study provides evidence for a central role of sqst-1 in HS-mediated homeostasis, illustrating a specific requirement for sqst-1 during proteotoxic stress. Moreover, overexpression of SQST-1 increased longevity and improved neuronal and intestinal proteostasis by inducing autophagy in $C$. elegans. [We note that while this paper was under revision, David Walker's group reported that adult overexpression of $\operatorname{Ref}(2) / \mathrm{p} 62$ in Drosophila similarly extends lifespan in an autophagydependent manner ${ }^{61}$.] While our data support previous observations showing that increasing p62 levels in neurodegenerative disease is beneficial in mice ${ }^{10}$, accumulation of p62 in mammals can be cytotoxic in some tissues, and can have cancerous effects under specific circumstances ${ }^{15,62}$. Indeed, mammalian p62 is a 
multifunctional protein involved in multiple cellular processes besides autophagy, such as several signal-transduction cascades, amino-acid sensing and the antioxidant response, that are mediated by additional protein domains not present in C. elegans SQST- ${ }^{63}$. Notably, the cancerous effects of p62 appear to be independent of the autophagy-related functions of $p 62^{62,64}$. Nevertheless, an increase in p62-mediated selective autophagy under cytotoxic conditions could be beneficial and possibly counteract age-related neurodegenerative diseases.

\section{Methods}

Strains. Strains were maintained and cultured under standard conditions at $20^{\circ} \mathrm{C}$ with the exception of autophagy measurements after hormetic heat shock, when animals were cultured at $15^{\circ} \mathrm{C}^{65}$. For experiments with temperature-sensitive $g l p-1$ (e2144) mutants, both glp-1(e2144) and wild-type (WT) N2 animals were allowed to hatch at $20^{\circ} \mathrm{C}$ for $24 \mathrm{~h}$ and then moved to $25^{\circ} \mathrm{C}$ until day 1 of adulthood. Escherichia coli OP50 was used as a food source unless RNAi was initiated. For RNAi experiments, animals were grown on HT115 bacteria from the time of RNAi initiation (see below). See Supplementary Data 4 for strains used in and created for this study.

RNA interference. Gene inactivation was achieved by feeding C. elegans with RNAi bacterial clones expressing double-stranded RNA (dsRNA) targeting the gene of interest. Clones were obtained from the Ahringer RNAi library (cup-5, epg5, $\operatorname{lgg}$-1/Atg8, vps-34, cyc-1, nuo-6) or the Vidal RNAi library (atg-18, bec-1/ATG6). The let-363 and daf-2 RNAi clones and the empty vector (L4440) for controls were provided by Drs. J. Avruch, A. Dillin, and A. Fire, respectively. All RNAi clones were verified by sequencing. For RNAi experiments, HT115 bacteria were grown in Luria-Bertani (LB) liquid culture medium containing $0.1 \mathrm{mg} \mathrm{ml}^{-1}$ carbenicillin (Carb; BioPioneer) and $80 \mu \mathrm{l}$ aliquots of bacterial suspension were spotted onto $6 \mathrm{~cm}$ nematode growth medium (NGM) containing $0.1 \mathrm{mg} \mathrm{ml}^{-1}$ Carb (NGM/Carb plates). Bacteria were allowed to grow for 1-2 days. For induction of dsRNA expression, $80 \mu \mathrm{l}$ of a solution containing $0.1 \mathrm{M}$ isopropyl-b-D-thiogalactoside (Promega/Gold Biotechnology) and $500 \mathrm{mg} \mathrm{ml}^{-1}$ Carb was placed directly onto the lawn. For two generations of RNAi, animals were synchronized by hypochlorous acid treatment or eggs were manually transferred onto NGM plates seeded with dsRNA-expressing HT115 bacteria, once these animals reached adulthood, their progeny was transferred onto NGM plates seeded with dsRNA-expressing HT115 bacteria. For whole-life RNAi, animals were synchronized by hypochlorous acid treatment or eggs were manually transferred onto NGM plates seeded with dsRNA-expressing HT115 bacteria. For adult-only RNAi, animals were synchronized by hypochlorous acid treatment and eggs were allowed to hatch on NGM plates seeded with OP50 bacteria. On day 1 of adulthood, animals were transferred to NGM/Carb plates seeded with dsRNA-expressing or control bacteria.

Autophagy measurements. Autophagy was monitored by counting GFP-positive LGG-1/Atg8 punctae in body-wall muscle, proximal intestinal cells, and terminal pharyngeal bulb of strain DA2123, MAH693, MAH925, MAH236, MAH864, RD202, and MAH711. Autophagy was monitored in the nerve-ring neurons of strain MAH242, MAH690, MAH944, MAH855, MAH856, MAH744, MAH779, MAH785, and MAH904. See Supplementary Data 4 for genotypes and strain information. For hormetic $\mathrm{HS}$ experiments, animals were raised at $15^{\circ} \mathrm{C}$ and subjected to $\mathrm{HS}$ for $1 \mathrm{~h}$ at $36^{\circ} \mathrm{C}$. For all other experiments, animals were raised at $20^{\circ} \mathrm{C}$.

For autophagic flux assays, bafilomycin Al (BafA) (BioViotica) or vehicle (dimethylsulfoxide, DMSO) was injected into gfp::lgg-1/ATG8-expressing animals on day 1 of adulthood ${ }^{40}$. BafA was resuspended in DMSO to a stock concentration of $25 \mu \mathrm{M}$. The co-injection dye Texas Red Dextran $3000 \mathrm{MW}$ (Molecular Probes) was resuspended in water to a stock concentration of $25 \mathrm{mg} \mathrm{ml}^{-1}$. A $100 \mu \mathrm{M} \mathrm{BafA}$ working solution was prepared by adding $1 \mu$ of the $25 \mathrm{mM}$ BafA stock solution to $249 \mu \mathrm{l}$ of water. The $50 \mu \mathrm{M}$ BafA injection solution was prepared by mixing $5 \mu \mathrm{l}$ of $100 \mu \mathrm{M}$ BafA with $1 \mu \mathrm{l}$ of the $25 \mathrm{mg} \mathrm{ml}^{-1}$ co-injection dye with $4 \mu \mathrm{l}$ of water. A $0.2 \%$ DMSO injection solution containing the co-injection dye was prepared the same way. The BafA or DMSO injection solution was injected into the anterior intestinal area or close to the terminal pharyngeal bulb and animals were allowed to recover on NGM plates with OP50 for $2 \mathrm{~h}$. Surviving animals that scored positive for the red dye were used for GFP::LGG-1 punctae quantification.

For imaging and punctae quantification, animals were mounted on a $2 \%$ agarose pad in $\mathrm{M} 9$ medium containing $0.1 \% \mathrm{NaN}_{3}$ and GFP::LGG-1/Atg8 punctae were counted using a Zeiss Imager Z1 including apotome.2 with a Hamamatsu orca flash 4LT camera and Zen 2.3 software. The total number of GFP::LGG-1/Atg8positive punctae was counted in body-wall muscle, the three to four most proximal intestinal cells or the terminal pharyngeal bulb at $\times 1000$ magnification. For imaging and punctae quantification of the nerve-ring neurons, Z-stack images were acquired at $1.0 \mu \mathrm{m}$ slice intervals. At least 8-10 animals were imaged for each condition and results were combined from three independent experiments and analyzed using Student's $t$-test, one-way analysis of variance (ANOVA) or two-way
ANOVA as applicable (GraphPad Prism). The size of autophagic structures was determined by tracing the outline of GFP::LGG-1/Atg8-positive punctae from acquired images using ImageJ. Histograms of diameters of autophagic structures binned by diameter were made using GraphPad Prism.

Thermorecovery assays. For each strain, four $6 \mathrm{~cm}$ NGM plates with $20-40$ animals per plate were incubated in a single layer in a HERAtherm incubator (ThermoFisher) at $36^{\circ} \mathrm{C}$ for $7 \mathrm{~h}$. Animal survival was measured after $7 \mathrm{~h}$ at $36^{\circ} \mathrm{C}$ followed by a 'recovery' period of $20 \mathrm{~h}$ at $20^{\circ} \mathrm{C}$. Survival was assessed by scoring the animals' voluntary movement. For hormetic HS, animals were incubated at $36^{\circ} \mathrm{C}$ for $1 \mathrm{~h}$ on day 1 of adulthood and incubated for $7 \mathrm{~h}$ at $36^{\circ} \mathrm{C}$ on day 3 of adulthood. The average percentage survival and s.e.m. were calculated and the data were analyzed by one-way analysis of variance (ANOVA) or two-way ANOVA as applicable (GraphPad Prism)

Lifespan analysis. Lifespan was measured at $20^{\circ} \mathrm{C}$ using six $6 \mathrm{~cm} \mathrm{NGM} \mathrm{or} \mathrm{NGM/}$ Carb plates seeded with OP50 bacteria or dsRNA-expressing bacteria with 15-20 animals per plate. The L4 larval stage was recorded as day 0 of the lifespan and animals were transferred at least every other day to new $6 \mathrm{~cm}$ NGM plates throughout the reproductive period. For RNAi experiments, feeding with dsRNAexpressing or control bacteria was initiated on day 1 of adulthood unless indicated otherwise. For hormetic HS, animals were incubated at $36^{\circ} \mathrm{C}$ for $1 \mathrm{~h}$ on day 1 of adulthood. Animals were scored as dead if they failed to respond to gentle prodding with a platinum-wire pick. Censoring occurred if animals desiccated on the edge of the plate, escaped, ruptured or suffered from internal hatching. Statistical analysis was performed using Stata software (StataCorp) or Oasis $2^{66} . P$-values were calculated with the log-rank (Mantel-Cox) method. See Supplementary Tables 2-5 and Supplementary Data 1-3 for a summary of all lifespan experiments. For methodology on healthspan parameters see Supplementary Methods.

Fluorescence recovery after photobleaching (FRAP). The mobility of PolyQ aggregates was assayed using FRAP on a Nikon A1R HD confocal microscope ${ }^{67}$ Animals were either subjected to a hormetic $\mathrm{HS}\left(36^{\circ} \mathrm{C}, 1 \mathrm{~h}\right)$ on day 1 of adulthood, or maintained at control conditions $\left(20^{\circ} \mathrm{C}\right)$. For FRAP of neuronal Q40::YFP, animals were aged until day 8 of adulthood, for FRAP of intestinal Q44::YFP and muscle Q35::YFP, animals on day 3 of adulthood were used. For FRAP Animals were anaesthetized with $\mathrm{M} 9$ medium containing $0.1 \% \mathrm{NaN}_{3}$ and aggregated and diffuse PolyQ::YFP was bleached with $100 \%$ power of a $405 \mathrm{~nm}$ laser for $3.6 \mathrm{~s}$ (neurons) or $5.83 \mathrm{~s}$ (muscle and intestine). Recovery was monitored for a duration of $2 \mathrm{~min}$, with measurements every $30 \mathrm{~s}$ (neurons) or every $10 \mathrm{~s}$ (muscle and intestine) with $1 \%$ of a $488 \mathrm{~nm}$ laser. Fluorescence intensity was relative to prebleach intensity and a non-bleached area as well as a non-fluorescent background was monitored and corrected for. At least 30 animals were imaged for each condition and results were combined from three independent experiments and analyzed using two-way ANOVA (GraphPad Prism).

Analysis of PolyQ strains and temperature-sensitive mutants. The number of neuronal PolyQ aggregates was counted in individual animals of strains AM101, MAH755, MAH876 and MAH877 ( $f+r$ on days 2-14 of adulthood. The number of intestinal PolyQ aggregates was counted in individual animals of strains MAH602 and MAH691 on days 2-5 of adulthood. The number of muscle PolyQ aggregates was counted in individual animals of strains AM140 and MAH745 on days 2-3 of adulthood. See Supplementary Data 4 for genotypes and strain information. Animals were raised and maintained at $20^{\circ} \mathrm{C}$. For hormetic HS animals were raised at $20^{\circ} \mathrm{C}$ and then maintained under control conditions or subjected to HS for $1 \mathrm{~h}$ at $36{ }^{\circ} \mathrm{C}$ on day 1 of adulthood. Animals were imaged on NGM plates without food after anaesthetization with $\mathrm{M} 9$ medium containing $0.1 \% \mathrm{NaN}_{3}$. Images were acquired with a Leica DFC310 FX camera. Aggregates were counted using the 'Cell counter' function of ImageJ software. Averages and s.e.m. were calculated and the data were analyzed by two-way ANOVA (GraphPad Prism). For methodology on paralysis measurements of temperature-sensitive mutants, see Supplementary Methods.

Imaging of fluorescent reporter strains. For images of higher magnification (including for colocalization of SQST-1::GFP and tdTOMATO::LGG-1/Atg8), animals were mounted on a $2 \%$ agarose pad in $\mathrm{M} 9$ medium containing $0.1 \% \mathrm{NaN}_{3}$ and imaged on a Zeiss Imager Z1 including apotome.2 with a Hamamatsu orca flash 4LT camera and Zen 2.3 software at $\times 1000$ magnification. For colocalization, images were analyzed using the colocalization plugin Coloc2 in Fiji-ImageJ (National Institutes of Health). The correlation between the red and green fluorescence is given as intensity correlation quotient (ICQ) values ${ }^{68}$. For random or mixed patterns of fluorescence intensity of the red and green channel this number will tend toward 0 , for segregated localization of the red and green fluorescence signal the ICQ score will tend toward -0.5 , and for colocalization of the fluorescent signals, the ICQ score will tend toward +0.5 .

Biochemical assays. For methodology on differential detergent extraction, western blot analysis, and proteasomal activity assays, see Supplementary Methods. 
Quantitative RT-PCR. For methodology on quantitative RT-PCR, see Supplementary Methods. For sequences of primers used for quantitative RT-PCR, see Supplementary Table 6.

Reporting summary. Further information on research design is available in the Nature Research Reporting Summary linked to this article.

\section{Data availability}

The authors declare that all data supporting the findings of this study are available within this article, its Supplementary Information files, the peer-review file, or are available from the corresponding author upon reasonable request.

Received: 22 November 2018; Accepted: 13 November 2019; Published online: 11 December 2019

\section{References}

1. Kroemer, G., Marino, G. \& Levine, B. Autophagy and the integrated stress response. Mol. Cell 40, 280-293 (2010).

2. Kumsta, C., Chang, J. T., Schmalz, J. \& Hansen, M. Hormetic heat stress and HSF-1 induce autophagy to improve survival and proteostasis in C. elegans. Nat. Commun. 8, 14337 (2017).

3. Hansen, M., Rubinsztein, D. C. \& Walker, D. W. Autophagy as a promoter of longevity: insights from model organisms. Nat. Rev. Mol. Cell Biol. 19, 579-593 (2018).

4. Gatica, D., Lahiri, V. \& Klionsky, D. J. Cargo recognition and degradation by selective autophagy. Nat. Cell Biol. 20, 233-242 (2018).

5. Pankiv, S. et al. p62/SQSTM1 binds directly to Atg8/LC3 to facilitate degradation of ubiquitinated protein aggregates by autophagy. J. Biol. Chem. 282, 24131-24145 (2007)

6. Ichimura, Y. et al. Structural basis for sorting mechanism of p62 in selective autophagy. J. Biol. Chem. 283, 22847-22857 (2008).

7. Bjorkoy, G. et al. p62/SQSTM1 forms protein aggregates degraded by autophagy and has a protective effect on huntingtin-induced cell death. J. Cell Biol. 171, 603-614 (2005).

8. Korolchuk, V. I., Menzies, F. M. \& Rubinsztein, D. C. Mechanisms of crosstalk between the ubiquitin-proteasome and autophagy-lysosome systems. FEBS Lett. 584, 1393-1398 (2010).

9. Liu, W. J. et al. p62 links the autophagy pathway and the ubiqutin-proteasome system upon ubiquitinated protein degradation. Cell Mol. Biol. Lett. 21, 29 (2016).

10. Caccamo, A., Ferreira, E., Branca, C. \& Oddo, S. p62 improves AD-like pathology by increasing autophagy. Mol. Psychiatry 22, 865-873 (2017).

11. Doi, H. et al. p62/SQSTM1 differentially removes the toxic mutant androgen receptor via autophagy and inclusion formation in a spinal and bulbar muscular atrophy mouse model. J. Neurosci. 33, 7710-7727 (2013).

12. Babu, J. R., Geetha, T. \& Wooten, M. W. Sequestosome 1/p62 shuttles polyubiquitinated tau for proteasomal degradation. J. Neurochem 94, 192-203 (2005).

13. Nezis, I. P. et al. $\operatorname{Ref}(2) \mathrm{P}$, the Drosophila melanogaster homologue of mammalian p62, is required for the formation of protein aggregates in adult brain. J. Cell Biol. 180, 1065-1071 (2008).

14. Tian, Y. et al. C. elegans screen identifies autophagy genes specific to multicellular organisms. Cell 141, 1042-1055 (2010).

15. Komatsu, M. et al. Homeostatic levels of p62 control cytoplasmic inclusion body formation in autophagy-deficient mice. Cell 131, 1149-1163 (2007).

16. Arrasate, M., Mitra, S., Schweitzer, E. S., Segal, M. R. \& Finkbeiner, S. Inclusion body formation reduces levels of mutant huntingtin and the risk of neuronal death. Nature 431, 805-810 (2004).

17. Caughey, B. \& Lansbury, P. T. Protofibrils, pores, fibrils, and neurodegeneration: separating the responsible protein aggregates from the innocent bystanders. Annu Rev. Neurosci. 26, 267-298 (2003).

18. Kopito, R. R. Aggresomes, inclusion bodies and protein aggregation. Trends Cell Biol. 10, 524-530 (2000).

19. Kwon, J. et al. Assurance of mitochondrial integrity and mammalian longevity by the p62-Keap1-Nrf2-Nqo1 cascade. EMBO Rep. 13, 150-156 (2012).

20. Ramesh Babu, J. et al. Genetic inactivation of p62 leads to accumulation of hyperphosphorylated tau and neurodegeneration. J. Neurochem. 106, 107-120 (2008).

21. Wooten, M. W. et al. Essential role of sequestosome 1/p62 in regulating accumulation of Lys63-ubiquitinated proteins. J. Biol. Chem. 283, 6783-6789 (2008).

22. de Castro, I. P. et al. Drosophila ref(2)P is required for the parkin-mediated suppression of mitochondrial dysfunction in pink1 mutants. Cell Death Dis. 4, e873 (2013).
23. Schiavi, A. et al. Iron-starvation-induced mitophagy mediates lifespan extension upon mitochondrial stress in C. elegans. Curr. Biol. 25, 1810-1822 (2015).

24. Lin, L. et al. The scaffold protein EPG-7 links cargo-receptor complexes with the autophagic assembly machinery. J. Cell Biol. 201, 113-129 (2013).

25. Du, Y., Wooten, M. C., Gearing, M. \& Wooten, M. W. Age-associated oxidative damage to the p62 promoter: implications for Alzheimer disease. Free Radic. Biol. Med. 46, 492-501 (2009).

26. Du, Y., Wooten, M. C. \& Wooten, M. W. Oxidative damage to the promoter region of SQSTM1/p62 is common to neurodegenerative disease. Neurobiol. Dis. 35, 302-310 (2009).

27. Richter, K., Haslbeck, M. \& Buchner, J. The heat shock response: life on the verge of death. Mol. Cell 40, 253-266 (2010).

28. Vabulas, R. M., Raychaudhuri, S., Hayer-Hartl, M. \& Hartl, F. U. Protein folding in the cytoplasm and the heat shock response. Cold Spring Harb. Perspect. Biol. 2, a004390 (2010).

29. Wallace, E. W. et al. Reversible, specific, active aggregates of endogenous proteins assemble upon heat stress. Cell 162, 1286-1298 (2015)

30. Salomons, F. A. et al. Selective accumulation of aggregation-prone proteasome substrates in response to proteotoxic stress. Mol. Cell Biol. 29, 1774-1785 (2009)

31. Guo, B. et al. The nascent polypeptide-associated complex is essential for autophagic flux. Autophagy 10, 1738-1748 (2014).

32. McKay, S. J. et al. Gene expression profiling of cells, tissues, and developmental stages of the nematode C. elegans. Cold Spring Harb. Symp. Quant. Biol. 68, 159-169 (2003).

33. Springhorn, A. \& Hoppe, T. Western blot analysis of the autophagosomal membrane protein LGG-1/LC3 in Caenorhabditis elegans. Methods Enzymol. 619, 319-336 (2019).

34. Melendez, A. et al. Autophagy genes are essential for dauer development and life-span extension in C. elegans. Science 301, 1387-1391 (2003).

35. Kang, C., You, Y. J. \& Avery, L. Dual roles of autophagy in the survival of Caenorhabditis elegans during starvation. Genes Dev. 21, 2161-2171 (2007).

36. Gelino, S. et al. Intestinal autophagy improves healthspan and longevity in $C$ elegans during dietary restriction. PLoS Genet. 12, e1006135 (2016).

37. Manil-Segalen, M. et al. The C. elegans LC3 acts downstream of GABARAP to degrade autophagosomes by interacting with the HOPS subunit VPS39. Dev. Cell 28, 43-55 (2014).

38. Klionsky, D. J. et al. Guidelines for the use and interpretation of assays for monitoring autophagy (3rd edition). Autophagy 12, 1-222 (2016).

39. Zhang, H. et al. Guidelines for monitoring autophagy in Caenorhabditis elegans. Autophagy 11, 9-27 (2015).

40. Wilkinson, D. S. et al. Phosphorylation of LC3 by the Hippo kinases STK3/ STK4 is essential for autophagy. Mol. cell 57, 55-68 (2015).

41. Morley, J. F., Brignull, H. R., Weyers, J. J. \& Morimoto, R. I. The threshold for polyglutamine-expansion protein aggregation and cellular toxicity is dynamic and influenced by aging in Caenorhabditis elegans. Proc. Natl Acad. Sci. USA 99, 10417-10422 (2002)

42. Mohri-Shiomi, A. \& Garsin, D. A. Insulin signaling and the heat shock response modulate protein homeostasis in the Caenorhabditis elegans intestine during infection. J. Biol. Chem. 283, 194-201 (2008).

43. Gidalevitz, T., Ben-Zvi, A., Ho, K. H., Brignull, H. R. \& Morimoto, R. I. Progressive disruption of cellular protein folding in models of polyglutamine diseases. Science 311, 1471-1474 (2006).

44. Moronetti Mazzeo, L. E., Dersh, D., Boccitto, M., Kalb, R. G. \& Lamitina, T. Stress and aging induce distinct polyQ protein aggregation states. Proc. Natl Acad. Sci. USA 109, 10587-10592 (2012).

45. Jia, K., Hart, A. C. \& Levine, B. Autophagy genes protect against disease caused by polyglutamine expansion proteins in Caenorhabditis elegans. Autophagy 3, 21-25 (2007).

46. Prahlad, V. \& Morimoto, R. I. Integrating the stress response: lessons for neurodegenerative diseases from C. elegans. Trends Cell Biol. 19, 52-61 (2009).

47. Ben-Zvi, A., Miller, E. A. \& Morimoto, R. I. Collapse of proteostasis represents an early molecular event in Caenorhabditis elegans aging. Proc. Natl Acad. Sci. USA 106, 14914-14919 (2009).

48. Vilchez, D. et al. RPN-6 determines C. elegans longevity under proteotoxic stress conditions. Nature 489, 263-268 (2012).

49. Sun, T., Wang, X., Lu, Q., Ren, H. \& Zhang, H. CUP-5, the C. elegans ortholog of the mammalian lysosomal channel protein MLN1/TRPML1, is required for proteolytic degradation in autolysosomes. Autophagy 7, 1308-1315 (2011)

50. Wu, Y. et al. PI3P phosphatase activity is required for autophagosome maturation and autolysosome formation. EMBO Rep. 15, 973-981 (2014).

51. Toth, M. L. et al. Longevity pathways converge on autophagy genes to regulate life span in Caenorhabditis elegans. Autophagy 4, 330-338 (2008).

52. Wu, F. et al. Structural basis of the differential function of the two C. elegans Atg8 homologs, LGG-1 and LGG-2, in autophagy. Mol. Cell 60, 914-929 (2015). 
53. Chang, J. T., Kumsta, C., Hellman, A. B., Adams, L. M. \& Hansen, M. Spatiotemporal regulation of autophagy during Caenorhabditis elegans aging. Elife 6, https://doi.org/10.7554/eLife.18459 (2017).

54. Ventura, N. et al. p53/CEP-1 increases or decreases lifespan, depending on level of mitochondrial bioenergetic stress. Aging Cell 8, 380-393 (2009).

55. Zaffagnini, G. \& Martens, S. Mechanisms of selective autophagy. J. Mol. Biol. 428, 1714-1724 (2016).

56. Itakura, E. \& Mizushima, N. p62 Targeting to the autophagosome formation site requires self-oligomerization but not LC3 binding. J. Cell Biol. 192, 17-27 (2011).

57. Fujita, N. et al. Recruitment of the autophagic machinery to endosomes during infection is mediated by ubiquitin. J. Cell Biol. 203, 115-128 (2013).

58. Sun, D., Wu, R., Zheng, J., Li, P. \& Yu, L. Polyubiquitin chain-induced 062 phase separation drives autophagic cargo segregation. Cell Res. 28, 405-415 (2018).

59. Xie, Z., Nair, U. \& Klionsky, D. J. Atg8 controls phagophore expansion during autophagosome formation. Mol. Biol. Cell 19, 3290-3298 (2008).

60. Zaffagnini, G. et al. p62 filaments capture and present ubiquitinated cargos for autophagy. EMBO J 37, https://doi.org/10.15252/embj.201798308 (2018).

61. Aparicio, R., Rana, A. \& Walker, D. W. Upregulation of the autophagy adaptor p62/SQSTM1 prolongs health and lifespan in middle-aged Drosophila. Cell Rep. 28, 1029-1040 (2019).

62. Moscat, J., Karin, M. \& Diaz-Meco, M. T. p62 in cancer: signaling adaptor beyond autophagy. Cell 167, 606-609 (2016).

63. Bitto, A. et al. P62/SQSTM1 at the interface of aging, autophagy, and disease. Age 36, 9626 (2014).

64. Umemura, A. et al. p62, upregulated during preneoplasia, induces hepatocellular carcinogenesis by maintaining survival of stressed HCCinitiating cells. Cancer Cell 29, 935-948 (2016).

65. Brenner, S. The genetics of Caenorhabditis elegans. Genetics 77, 71-94 (1974).

66. Han, S. K. et al. OASIS 2: online application for survival analysis 2 with features for the analysis of maximal lifespan and healthspan in aging research. Oncotarget 7, 56147-56152 (2016).

67. Prahlad, V. \& Morimoto, R. I. Neuronal circuitry regulates the response of Caenorhabditis elegans to misfolded proteins. Proc. Natl Acad. Sci. USA 108, 14204-14209 (2011).

68. Li, Q. et al. A syntaxin 1, Galpha(o), and N-type calcium channel complex at a presynaptic nerve terminal: analysis by quantitative immunocolocalization. $J$. Neurosci. 24, 4070-4081 (2004).

\section{Acknowledgements}

We thank Dr. Andrew Hellman, Andrew Davis, Linnea Adams, Reza Badiee, Kylie Hooker, Leslie Boyd, and the Sanford Burnham Prebys Imaging Core for technical assistance; Stanislav Kumsta for help with data analysis; and Drs. Renaud Legouis, Hong Zhang, Kang Shen, Alicia Melendez, Cynthia Kenyon, Roger Tsien, Andrew Fire, Erik Jorgensen, and Andrew Dillin for kindly providing reagents. Some of the nematode strains used in this work were provided by the Caenorhabditis Genetics Center (University of Minnesota), which is supported by the NIH-Office of Research Infra-structure
Program (P40 OD010440). This work was funded by the Deutsche Forschungsgemeinschaft and the European Research Council (consolidator grant 616499) (to T.H.), the Julie Martin Mid-Career Award in Aging Research from The Ellison Medical Foundation/AFAR (to M.H.), and by NIH grants AG058038 (to C.K.), and AG028664 (to M.H.).

\section{Author contributions}

C.K., J.T.C., D.V., and M.H. designed the experiments. J.T.C. and E.P.T. constructed strains. C.K., J.T.C., Re.L., E.P.T., Y.Y., E.C., S.L., and M.H. performed lifespan experiments. J.T.C., Re.L., E.C., S.L., and M.H. contributed to healthspan experiments. C.K. and Re.L. performed stress assays, aggregation assays and western blots. Ru.L. and I.S.M. performed proteostasis experiments. C.K. performed all other experiments. C.K. and M. H. wrote the paper, with input from coauthors. The SQST-1 antibody was provided by A. S. and T.H. ahead of publication ${ }^{33}$.

\section{Competing interests}

The authors declare no competing interests.

\section{Additional information}

Supplementary information is available for this paper at https://doi.org/10.1038/s41467 019-13540-4.

Correspondence and requests for materials should be addressed to C.K. or M.H.

Peer review information Nature Communications thanks Ehud Cohen and the other, anonymous, reviewer(s) for their contribution to the peer review of this work. Peer reviewer reports are available.

Reprints and permission information is available at http://www.nature.com/reprints

Publisher's note Springer Nature remains neutral with regard to jurisdictional claims in published maps and institutional affiliations.

Open Access This article is licensed under a Creative Commons Attribution 4.0 International License, which permits use, sharing, adaptation, distribution and reproduction in any medium or format, as long as you give appropriate credit to the original author(s) and the source, provide a link to the Creative Commons license, and indicate if changes were made. The images or other third party material in this article are included in the article's Creative Commons license, unless indicated otherwise in a credit line to the material. If material is not included in the article's Creative Commons license and your intended use is not permitted by statutory regulation or exceeds the permitted use, you will need to obtain permission directly from the copyright holder. To view a copy of this license, visit http://creativecommons.org/ licenses/by/4.0/.

(C) The Author(s) 2019 